\title{
HUT Observations of the Lyman Limit in AGN: Implications for Unified Models
}

\author{
G. Kriss, J. Krolik, J. Grimes, Z. Tsvetanov, B. Espey, W. Zheng, and \\ A. Davidsen \\ Department of Physics and Astronomy, The Johns Hopkins University, \\ Baltimore, $M D$ 21218, USA
}

\begin{abstract}
We observed a total of 13 low-redshift AGN with the Hopkins Ultraviolet Telescope (HUT) during the course of the Astro-1 and Astro- 2 missions. Of these, 4 show intrinsic Lyman-limit absorption NGC 1068, NGC 4151, NGC 3516 and NGC 3227. All galaxies with optically thick Lyman limits have extended narrow-line regions with bi-polar morphologies. All AGN with no absorption are Seyfert 1s with compact NLRs. These observations support geometrical shadowing as the means for collimating the ionizing radiation in unified models of AGN, most likely in a photoionized atmosphere above the obscuring torus.
\end{abstract}

\section{Introduction}

An advantage of being one of the last speakers is that my preceding colleagues have given you an excellent introduction. So, I need only remind you of a few key points. The most important feature to remember from Wei Zheng's (this volume) description of $H U T$ is its sensitivity below $1200 \AA$. This allows us to see the Lyman-limit region in low-redshift AGN. A glimpse of the far-ultraviolet properties of these bright, nearby AGN is important because our whole concept of a unified model is based on low-redshift objects. Nearby AGN are bright, allowing spectropolarimetry and high-resolution X-ray spectroscopy, and the good angular scale allows us to resolve the narrow-line region (NLR) with $H S T$ and from the ground.

Unified models of AGN use a combination of obscuration, reflection, and orientation to explain the different appearances of Seyfert 1 and Seyfert 2 galaxies (see the review by Antonucci 1993). This picture implies some anisotropy for the escaping ionizing radiation. As Wilson shows (this volume), this has important consequences for the morphology of the NLR. If NLR gas is spherically distributed about the nuclear region, the escaping radiation will produce biconical structures projected onto the sky in obscured AGN (Type 2) and more compact, more symmetric structures in those viewed pole on (Type 1s). By and large, this is what is observed (e.g., Pogge 1989; Evans et al. 1994).

In more general terms, one could classify the means of collimating the ionizing radiation in one of two categories: intrinsic anisotropy, or geometrical shadowing. Examples of intrinsically anisotropic radiation would be that due to relativistic beaming or the radiation from an accretion funnel in a geometrically 


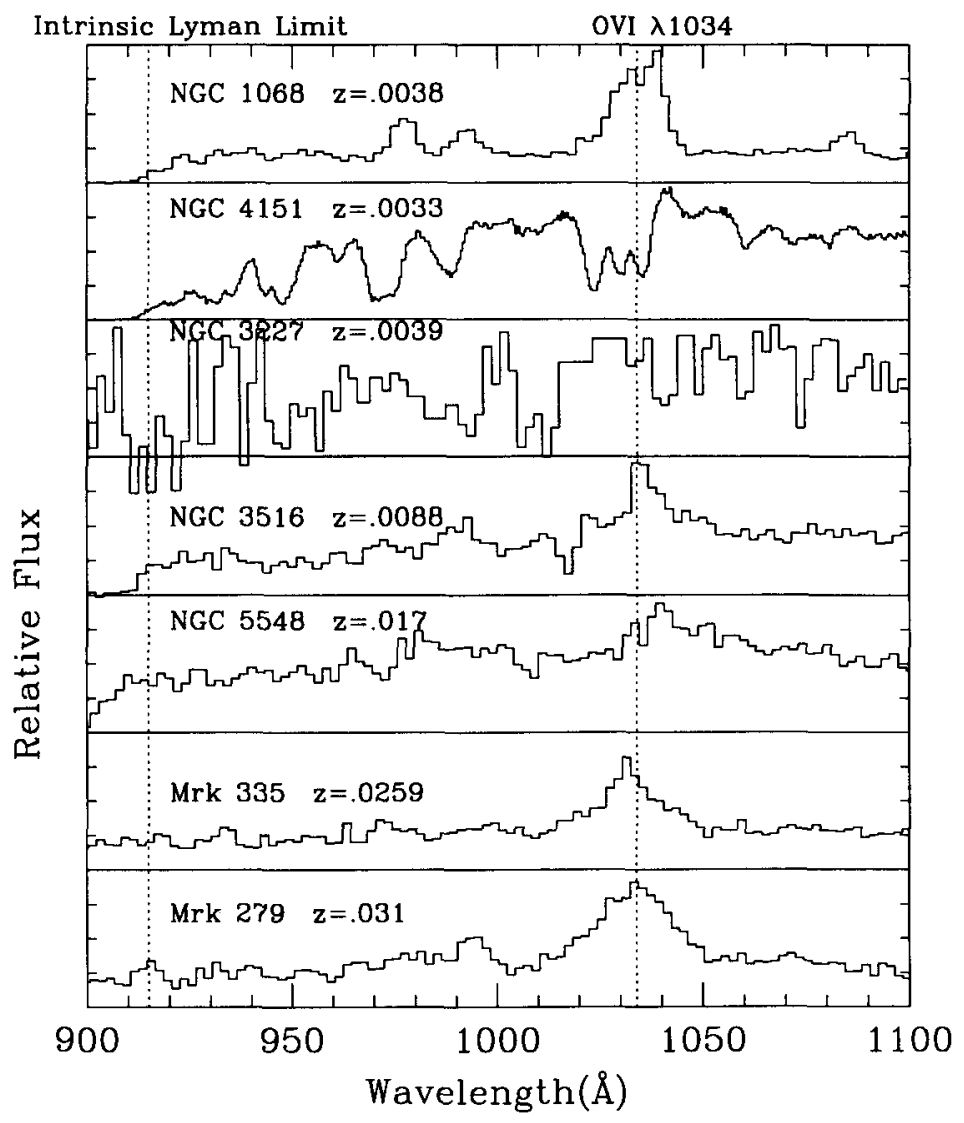

Figure 1. Representative $H U T$ spectra of the Lyman-limit region in low-redshift AGN. All spectra are shown in the object's rest frame.

thick accretion disk. Geometrical shadows that collimate the radiation can be produced not only by the obscuring torus, but also by the wind from an accretion disk or by a toroidal configuration of broad-line region clouds. All examples of shadowing predict we should see optically thick neutral-hydrogen absorption at the Lyman limit.

\section{HUT Observations}

Of 13 AGN with $z<0.3$ observed with $H U T$, only 4 show intrinsic Lyman limits. Table 1 summarizes the HUT Astro- 1 and Astro- 2 observations, and Fig. 1 shows representative spectra. All objects with Lyman-limit absorption (NGC 1068, NGC 4151, NGC 3516, and NGC 3227) have narrow-line regions with bipolar morphologies. The remaining 9 AGN are Seyfert $1 \mathrm{~s}$ or low-redshift quasars with point-like NLRs as imaged with HST or from the ground. Seeing 
absorption only in objects with bipolar NLRs favors shadowing for collimation over an intrinsically beamed mechanism.

Table 1. AGN with HUT Lyman-Limit Observations.

\begin{tabular}{lcccc}
\hline \multicolumn{1}{c}{ Object } & Type & $z$ & NLR Morphology & Lyman Limit? \\
\hline NGC 1068 & 2 & 0.0038 & bi-cone & yes \\
NGC 4151 & 1 & 0.0033 & bi-cone & yes \\
NGC 3227 & 1 & 0.0039 & bi-cone & yes (95\% confidence) \\
NGC 3516 & 1 & 0.0088 & S-shape & yes \\
NGC 5548 & 1 & 0.017 & point-like & no \\
Mrk 335 & 1 & 0.026 & point-like & no \\
Mrk 279 & 1 & 0.031 & point-like & no \\
Mrk 509 & 1 & 0.035 & point-like & no \\
Mrk 478 & 1 & 0.077 & point-like & no \\
PG 1211+143 & 1 & 0.081 & $?$ & no \\
PG 1351+640 & 1 & 0.088 & $?$ & no \\
3C 273 & 1 & 0.158 & jet & no \\
E 1821+64 & 1 & 0.297 & point-like & no \\
\hline
\end{tabular}

\section{The Location of the Collimator}

The next question is, if obscuration collimates the light, can we pinpoint the location of the absorbing gas? Monitoring variability in absorption features allows one to measure two time scales-the ionization time scale and the recombination time scale - each of which allows some measure of the distance of the absorbing gas from the central source. To apply this technique, we observed NGC 4151 with HUT 6 times at intervals of 2-3 days during the Astro- 2 mission, and we got lucky. Our data (Fig. 2) show an approximate $\delta$-function blip in the continuum flux and the change of the neutral hydrogen column in response. When the continuum flux rises (a $60 \%$ increase, or $\Delta L=1.4 \times 10^{43} \mathrm{ergs} \mathrm{s}^{-1}$ ), we see a nearly instantaneous drop in the neutral hydrogen column $\left(\Delta N(\mathrm{H})=7.5 \times 10^{17} \mathrm{~cm}^{-2}\right)$ giving an ionization time scale of $<2$ days, the limit of our sampling. After the continuum returns to its mean intensity, the neutral hydrogen column gradually increases with an $e$-folding time scale of $\sim 10$ days.

The decrease in the neutral hydrogen column $\Delta N(\mathrm{HI})$ and the time $\Delta t$ required to respond to the continuum increase $\Delta L$ provide a direct measure of the number of ionizing photons and indirectly give a measure of the distance of the absorber from the source. Since $\Delta N(\mathrm{HI})=$ \# ionizing photons $/ 4 \pi r^{2}$, and $\Delta L=$ (\# ionizing photons) $\times h \bar{\nu} / \Delta t$, the distance of the absorber from the source can be found as $r=[\Delta L \Delta t / 4 \pi \Delta N(\mathrm{HI}) h \bar{\nu}]^{1 / 2}$. For our observations of NGC 4151, an upper limit of 2 days on the ionization time scale translates into an upper limit of $30 \mathrm{pc}$ on the radius of the absorbing gas. 


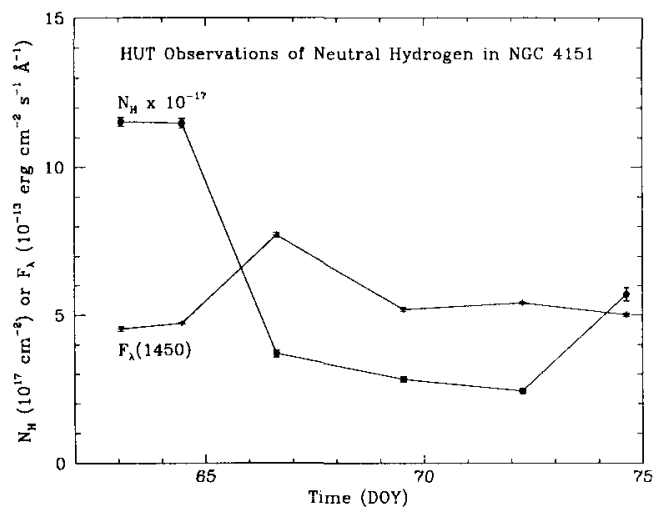

Figure 2. The variation in the neutral hydrogen column from $H U T$ observations of NGC 4151 during Astro-2 is shown along with the continuum intensity at $1450 \AA$. The $x$-axis is in day of the year 1995. Error bars, barely visible around each data point, are $1 \sigma$.

The recombination time scale, $t_{r e c}=\left(1 / n_{e} \alpha_{r e c}\right)[n(\mathrm{HI}) / n(\mathrm{H} \mathrm{II})]$, is often cited as a measure of the density of absorbing material, but this is true only if one knows the ionization state of the gas (Krolik \& Kriss 1995). The neutral fraction $n(\mathrm{HI}) / n(\mathrm{H} \mathrm{II})$ depends on the ionization parameter $U=L / 4 \pi r^{2} h \bar{\nu}\left(n_{e}+n_{p}\right)$ as $n(\mathrm{H} \mathrm{I}) / n(\mathrm{H} \mathrm{II})=Q / U$, where $Q$ is typically $\sim 4.5 \times 10^{-6}$, determined by atomic data and the shape of the ionizing spectrum. These relations can be used to obtain a distance estimate for the gas based on the observed recombination time:

$r=62 \mathrm{pc}\left(\frac{\Delta t}{10 \mathrm{~d}}\right)^{1 / 2}\left(\frac{L}{2.3 \times 10^{43} \mathrm{erg} \mathrm{s}^{-1}}\right)^{1 / 2}\left(\frac{15,000 \mathrm{~K}}{T}\right)^{0.4}\left(\frac{4.5 \times 10^{-6}}{Q}\right)^{1 / 2}$.

For our observations of NGC 4151, the absorbing gas is located far from the central source in the NLR. The absorbing gas may be outflowing material driven off the surface of the obscuring torus.

Given the limited sampling and the simplified treatment shown here, I would view these numbers as only order-of-magnitude estimates. They show, however, that the the absorbing gas is likely to be far from the central source, at or beyond the location of the obscuring torus. Our observations illustrate the power of monitoring absorption variability, and this technique offers real possibilities for learning more about both UV and X-ray absorbing gas.

Acknowledgments. This work was supported by NASA Contract NAS 5-27000 and NASA LTSA grant NAGW-4443 to the Johns Hopkins University.

\section{References}

Antonucci, R. 1993, Ann. Rev. Astr. Ap., 31, 473.

Evans, I., et al. 1994, ASP Conf. Ser., 54, 3.

Krolik, J.H., \& Kriss, G. A. 1995, ApJ, 447, 512.

Pogge, R. 1989, ApJ, 345, 730. 